\title{
AVRIL 1962
}

\section{REVUE}

\section{INTERNATIONALE}

\author{
DE LA
}

CROIX-ROUGE

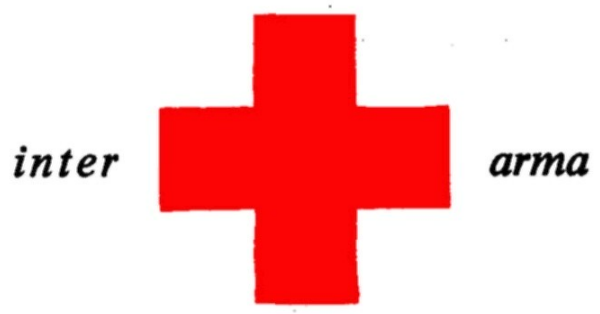

caritas 


\section{COMITÉ INTERNATIONAL DE LA CROIX-ROUGE}

MM. LEOPOLD BOISSIER, docteur en droit, professeur honoraire de l'Université de Genève, ancien secrétaire général de l'Union interparlementaire, président (membre depuis 1946)

JACQUES CHENEVIÈRE, docteur ès lettres h.c., vice-président d'honncur (1919).

CARL J. BURCKHARDT, docteur en philosophie, ancien ministre de Suisse en France (1933)

MARTIN BODMER, docteur en philosophie h.c. (1940)

ERNEST GLOOR, mèdecin, vice-présidem (1945)

PAUL RUEGGER, ancien ministre de Suisse en Italie et en Grande-Bretagne, membre de la Cour Permanente d'Arbitrage (1948), en congé

RODOLFO OLGIATI, docteur en médecine h.c., ancien directeur du Don Suisse (1949)

Mlle MARGUERITE VAN BERCHEM, ancien chef de service de l'Agence centrale des prisonniers de guerre (1951)

MM. FRÉDÉRIC SIORDET, avocat, conseiller du Comité international de la Croix-Rouge de 1943 à 1951, vice-président (1951)

GUILLAUME BORDIER, ingénieur dipl. E.P.F., M.B.A. Harvard, banquier (1955).

ADOLPHE FRANCESCHETTI, docteur en médecine, professeur de clinique ophtalmologique à l'Université de Genève (1958)

HANS BACHMANN, docteur en droit, secrétaire général adjoint du Comité international de la Croix-Rouge de 1944 à 1946 (1958)

JACQUES FREYMOND, docteur ès lettres, directeur de l'Institut universitaire de hautes études internationales, professeur à l'Université de Genève (1959)

DIETRICH SCHINDLER, docteur en droit (1961)

SAMUEL GONARD, ancien colonel commandant de corps, ancien professeur à l'Ecole polytechnique fédérale (1961)

HANS MEULI, docteur en médecine, colonel brigadier, ancien chef du Service de santé de l'armée suisse (1961)

M" MARJORIE DUVILLARD, directrice de l'école d'infirmières "Le Bon Secours" (1961)

M. MAX PETITPIERRE, docteur en droit, ancien président de la Confédération suisse (1961)

Membres honoraires: MHe LUCIE ODIER, vice-présidente d'honneur; MM. FREDERIC BARBEY et PAUL CARRY, $M^{\text {He }}$ SUZANNE FERRIĖRE, $M^{\text {me }}$ R. M. FRICK-CRAMER, MM. EDOUARD de HALLER, PAUL LOGOZ, ALFREDO VANNOTTI, ADOLF VISCHER.

Direction: MM. ROGER GALLOPIN, docteur en droit, directeur exécutif JEAN S. PICTET, docteur en droit, directeur des Affaires genérales EDOUARD de BONDELI, directeur financier et administratif CLAUDE PILLOUD, directeur-adjoint des Affaires générales. 


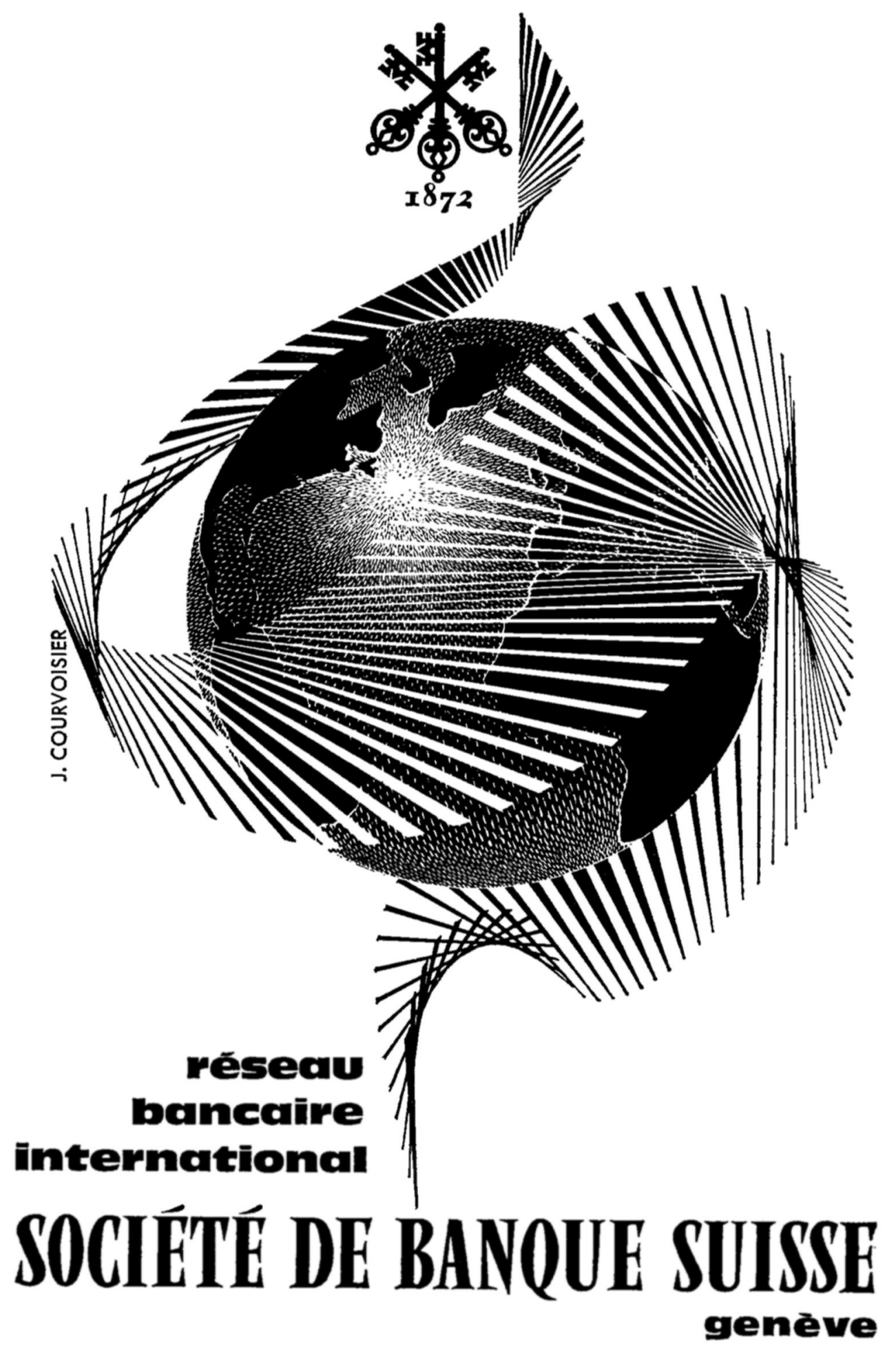




\section{SOCIETE FIDUCIAIRE ROMANDE OFOR S.A.}

9, rue d'Italie, Genève - Tél. 243293

- Expertises, revisions et organisations comptables

- Domiciliation et administration de sociétés

- Assainissements et liquidations

- Interventions et conseils en matière fiscale

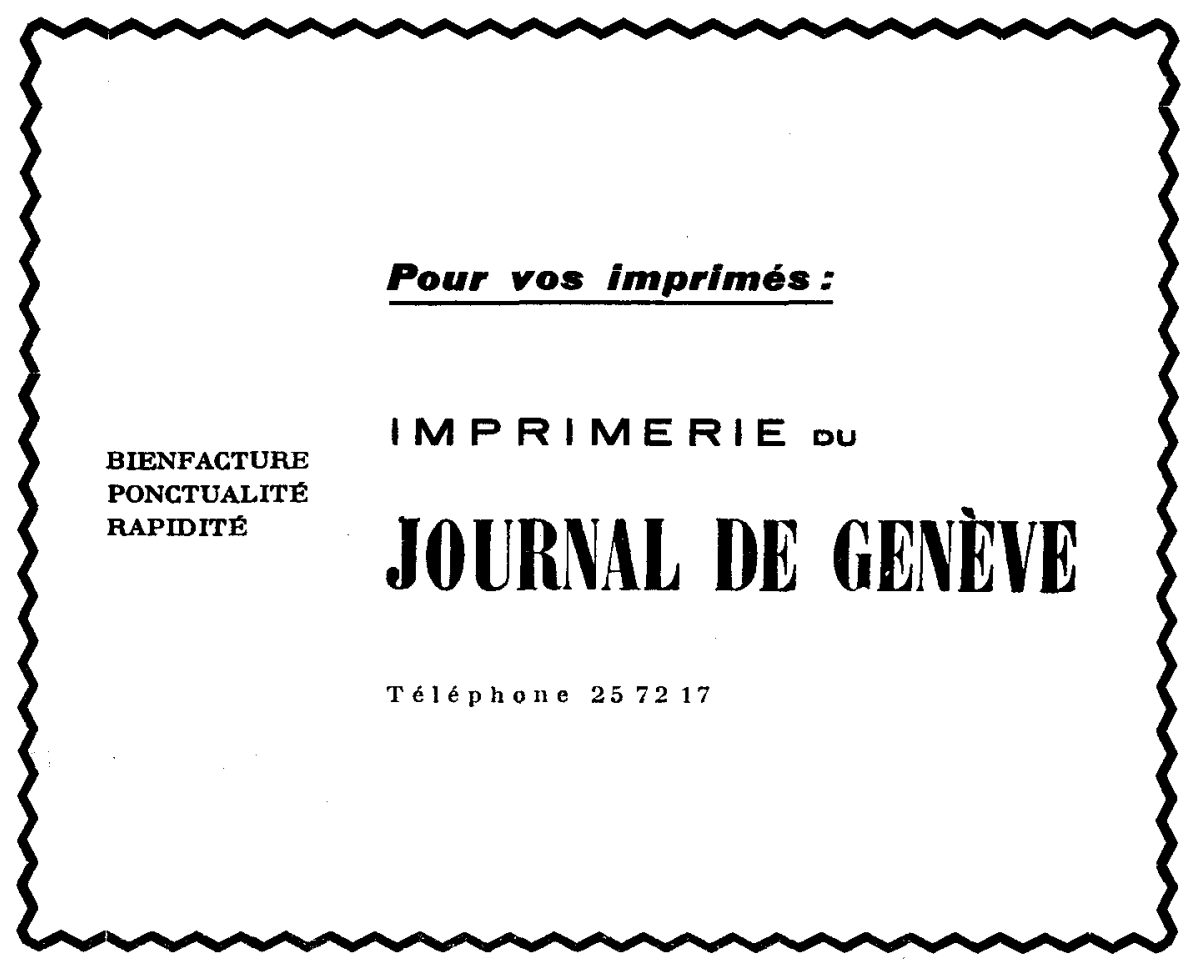




\section{REVUE INTERNATIONALE \\ DE LA CROIX - ROUGE}

QUARANTE-QUATRIEME ANNEE - No 520

$A V R I L \quad 1962$

\section{SOMMAIRE}

J.-G. Lossier : En visitant Castiglione et son Musée international de la Croix-Rouge

Jages I 65

\section{COMITÉ INTERNATIONAL DE LA CROIX-ROUGE}

Nouvelles d'ici et de partout . . . . . . . . . . . . . . . . I76

Visite à Rome du Président du Comité international . . . . . . I86

Les nouveaux Etats africains et les Conventions de Genève . . . I88

Conventions de Genève . . . . . . . . . . . . . . . . . . . Igo

Fonds de l'Impératrice Shôken (quarante et unième distribution

des revenus) . . . . . . . . . . . . . . . . . . . . $\mathrm{rgI}$

\section{NOUVELLES DE SOCIÉTÉS NATIONALES}

Espagne . . . . . . . . . . . . . . . . . . . . I94

Etats-Unis . . . . . . . . . . . . . . . . . . . . 197

\section{FAITS ET DOCUMENTS}

La santé mentale . . . . . . . . . . . . . . . . . . . . . 202

Personnel médical auxiliaire . . . . . . . . . . . . . . . . 205

Réadaptation des lépreux. . . . . . . . . . . . . . . . . 206

\section{BIBLIOGRAPHIE}

\section{A TRAVERS LES REVUES}




\section{EDITION ANGLAISE DE LA REVUE}

Une édition en langue anglaise paraît chaque mois sous le titre:

\section{International Review of the Red Cross}

Elle est en principe identique à l'édition française, et peut être obtenue aux mêmes conditions.

\section{SUPPLEMENTS DE LA REVUE}

\section{EN LANGUE ESPAGNOLE}

J.-G. Lossier: Una visita a Castiglione y a su Museo internacional de la Cruz Roja. - Visita a Roma del Presidente del Comité Internacional. - $i$ Estan siempre los archivos de la Primera Guerra Mundial útiles?. - Los nuevos Estados africanos y los Convenios de Ginebra.

\section{EN LANGUE ALIEMANDE}

J.-G. Lossier: Besuch in Castiglione und seinem Internationalen Rotkreuzmuseum. - Rombesuch des Präsidenten des Internationalen Komitees. - Sind die Archive des Ersten Weltkrieges immer noch von Nutzen?. - Die neuen afrikanischen Staaten und die Genfer Abkommen.

\section{La}

REVUE INTERNATIONALE DE LA CROIX-ROUGE est publiée chaque mois par le Comité international de la Croix-Rouge

7, avenue de la Paix, Genève (Suisse) - Compte de chèques postaux $I, 1767$

Abonnement un an: Fr. 20,-: le numéro Fr. 2,-

Pour les pays suivants: Algérie, Allemagne, Autriche, Belgique, Danemark, Finlande, France, Italie, Luxembourg, Maroc, Norvege, Pays-Bas, Portugal, Sarre, Suede, Tunisie, Vatican, on peut s'abonner auprès des bureaux de poste (fr. s. 20,50)

Rédaction: JEAN-G. LOSSIER 